\title{
MEASURES OF CHAOS AND A SPECTRAL DECOMPOSITION OF DYNAMICAL SYSTEMS ON THE INTERVAL
}

\author{
B. SCHWEIZER AND J. SMÍTAL
}

To our colleague and friend Abe Sklar on the occasion of his 65th birthday

\begin{abstract}
Let $f:[0,1] \rightarrow[0,1]$ be continuous. For $x, y \in[0,1]$, the upper and lower (distance) distribution functions, $F_{x y}^{*}$ and $F_{x y}$, are defined for any $t \geq 0$ as the limsup and liminf as $n \rightarrow \infty$ of the average number of times that the distance $\left|f^{i}(x)-f^{i}(y)\right|$ between the trajectories of $x$ and $y$ is less than $t$ during the first $n$ iterations. The spectrum of $f$ is the system $\Sigma(f)$ of lower distribution functions which is characterized by the following properties: (1) The elements of $\Sigma(f)$ are mutually incomparable; (2) for any $F \in \Sigma(f)$, there is a perfect set $P_{F} \neq \varnothing$ such that $F_{u v}=F$ and $F_{u v}^{*} \equiv 1$ for any distinct $u, v \in P_{F} ;(3)$ if $S$ is a scrambled set for $f$, then there are $F, G$ in $\Sigma(f)$ and a decomposition $S=S_{F} \cup S_{G}$ ( $S_{G}$ may be empty) such that $F_{u v} \geq F$ if $u, v \in S_{F}$ and $F_{u v} \geq G$ if $u, v \in S_{G}$. Our principal results are: (1) If $f$ has positive topological entropy, then $\Sigma(f)$ is nonempty and finite, and any $F \in \Sigma(f)$ is zero on an interval $[0, \varepsilon]$, where $\varepsilon>0$ (and hence any $P_{F}$ is a scrambled set in the sense of $\mathrm{Li}$ and Yorke). (2) If $f$ has zero topological entropy, then $\Sigma(f)=\{F\}$ where $F \equiv 1$.

It follows that the spectrum of $f$ provides a measure of the degree of chaos of $f$. In addition, a useful numerical measure is the largest of the numbers $\int_{0}^{1}(1-F(t)) d t$, where $F \in \Sigma(f)$.
\end{abstract}

\section{INTRODUCTION}

Let $I$ denote the unit interval $[0,1]$ and $C(I, I)$ the space of continuous functions from $I$ into $I$, endowed with the uniform metric. For any $f$ in $C(I, I)$ and any integer $i \geq 0$, let $f^{i}$ denote the $i$ th iterate of $f$. For any $x$ in $I$, the sequence of iterates $\left\{f^{i}(x)\right\}_{i=0}^{\infty}$, where $f^{0}(x)=x$, is the trajectory of $x$; and the set $\omega_{f}(x)$ of limit points of this trajectory is the $\omega$-limit set of $x$. A subset $J$ of $I$ is periodic with period $n$ if $f^{n}(J)=J$ and $f^{i}(J) \neq J$ for $1 \leq i<n$; and $\operatorname{Orb}(J)=J \cup f(J) \cup \cdots \cup f^{n-1}(J)$ is the orbit of $J$. A point $x$ in $I$ is periodic if the set $\{x\}$ is periodic. For any $x, y$ in $I$ and any $i \geq 0$, let $\delta_{x y}(i)$ denote the distance $\left|f^{i}(x)-f^{i}(y)\right|$ between the $i$ th iterates of $x$ and $y$. Lastly, for $\varepsilon>0$, a subset $S$ of $I$ is an $\varepsilon$-scrambled set (or, briefly, a scrambled set) for $f$ if, for distinct $x, y$ in $S$,

$$
\limsup _{i \rightarrow \infty} \delta_{x y}(i) \geq \varepsilon \text { and } \liminf _{i \rightarrow \infty} \delta_{x y}(i)=0 .
$$

Received by the editors November 16, 1992 and, in revised form, July 21, 1993.

1991 Mathematics Subject Classification. Primary 58F13, 58F08, 26A18; Secondary 54E70, $54 \mathrm{H} 20$.

Research supported by ONR contract N-00014-87-K-0379. 
Various notions of chaos are based on the behavior of sequences of distances $\left\{\delta_{x y}(i)\right\}_{i=0}^{\infty}$ between trajectories of distinct points $x, y$ in $I$. Thus a function $f$ is chaotic in the sense of Li and Yorke if there are two points $x, y$ in $I$ such that (1) is true for some $\varepsilon>0$ [9 and 7]. As shown in [19], this is equivalent to the condition that, for some $\varepsilon>0, f$ has a nonempty, perfect $\varepsilon$-scrambled set. (Note: We omit the requirement from [9] or [19] that $\lim _{\sup _{i \rightarrow \infty}} \delta_{x p}(i)>0$ for any periodic point $p$ of $f$ and any $x$ in the scrambled set since it is not essential, cf. [4].) Another notion of chaos is based on the topological entropy $h(f)$ of $f$, which is an asymptotic estimate of the size of maximal sets $A_{\varepsilon}$ such that $\sup _{i} \delta_{x y}(i)>\varepsilon$ for any distinct $x, y$ in $A_{\varepsilon}$ (cf. [2]). In this context, a function $f$ in $C(I, I)$ is considered to be chaotic if $h(f)>0$. Every function with $h(f)>0$ is chaotic in the sense of $\mathrm{Li}$ and Yorke. The converse is false (see [11 and 19] for details).

The sequences of distances $\left\{\delta_{x y}(i)\right\}_{i=0}^{\infty}$ have also been studied in the theory of probabilistic metric spaces [18]. But there the focus has been, not on these distances themselves, but rather on the limiting behavior as $n \rightarrow \infty$ of the distribution of their averages over the first $n$ iterations. Specifically, for any $f$ in $C(I, I)$, any $x, y$ in $I$, any real $t$, and any positive integer $n$, let

$$
\begin{aligned}
\xi(x, y, t, n) & =\sum_{i=0}^{n-1} \chi_{[0, t)}\left(\left|f^{i}(x)-f^{i}(y)\right|\right) \\
& =\#\left\{i ; 0 \leq i \leq n-1 \text { and } \delta_{x y}(i)<t\right\}
\end{aligned}
$$

let

$$
F_{x y}^{*}(t)=\limsup _{n \rightarrow \infty} \frac{1}{n} \xi(x, y, t, n)
$$

and let

$$
F_{x y}(t)=\liminf _{n \rightarrow \infty} \frac{1}{n} \xi(x, y, t, n) .
$$

Both $F_{x y}^{*}$ and $F_{x y}$ are nondecreasing functions and may be viewed as cumulative probability distribution functions satisfying $F_{x y}^{*}(t)=F_{x y}(t)=0$ for $t<0$ and $F_{x y}^{*}(t)=F_{x y}(t)=1$ for $t>1$. Any two such functions that coincide everywhere except at a countable set of points (of discontinuity) are identified; accordingly, the space of these functions will be equipped with the topology induced by the $L^{1}$ metric or, equivalently (since $I$ is bounded), with the topology of weak convergence of distribution functions. In general we will follow the convention adopted in [18] and choose $F_{x y}^{*}$ and $F_{x y}$ to be left-continuous. These functions are, respectively, the upper and lower (distance) distribution functions of $x$ and $y$. As discussed in [18, Chapter 11], where further references to the literature may be found, the lower distribution functions always determine a probabilistic pseudometric on $I$. Furthermore, if $f$ has an invariant probability measure $\mu$, then for almost all pairs $(x, y)$ in $I^{2}, F_{x y}=F_{x y}^{*}$; and if, in addition, $f$ is mixing with respect to $\mu$, then $F_{x y}$ is almost everywhere independent of the initial points $x, y$.

The study of the distribution functions $F_{x y}$ and $F_{x y}^{*}$ determined by a given $f$ in $C(I, I)$ is the central topic of our paper. However, in our situation, while the central idea is of paramount importance, neither the above-mentioned results nor the techniques used to obtain them are applicable. The principal 
reason for this is that, typically, i.e., except for a set of the first Baire category in $C(I, I)$, the functions we consider have only singular invariant measures supported by sets of zero Lebesgue measure. In such cases, the results based on the existence of invariant measures say nothing about the behavior of trajectories starting from sets of full Lebesgue measure. Consequently, we will adopt a direct approach and deal explicitly with the upper and lower distributions generated by a given $f$. It will follow from our results that the set of these distributions can be used as a measure of chaos.

To illustrate our ideas with a simple example, consider the standard "tent" map $g: I \rightarrow I$ which is given by $g(x)=1-|2 x-1|$. This map has positive topological entropy and there is exactly one maximal $\omega$-limit set, namely $I$ itself. In this case we find that there exist points $u, v$ for which $F_{u v}<F_{u v}^{*}$ and which are such that

$$
F_{u v} \leq F_{x y} \leq F_{x y}^{*} \leq F_{u v}^{*} \equiv 1
$$

for all pairs of points $x, y$ in $I$. Thus $F_{u v}$ and $F_{u v}^{*}$ are extremal distributions for $g$. As indicated, $F_{u v}^{*}$ is identically equal to 1 . The lower distribution $F_{u v}$ can also be explicitly determined: we find that $F_{u v}$ is the discrete distribution given by $F_{u v}(0)=0$ and

$$
F_{u v}(x)=1-\frac{1}{n} \text { for } x \in\left(a_{n-1}, a_{n}\right],
$$

where $a_{0}=0$ and $a_{n}=2^{n} /\left(2^{n}+1\right)$ for $n=1,2, \ldots$. Note that $a_{n}$ is the largest periodic point of period $n$ of $g$. Moreover, since the tent map is mixing with respect to Lebesgue measure on $I$, the limiting distribution function $F_{x y}=F_{x y}^{*}=F$ exists and is independent of $x, y$, for almost all pairs of points $(x, y)$ in $I^{2}$ (as already mentioned): it is given explicitly by $F(t)=2 t-t^{2}$ for $t \in[0,1][18$, Chapter 11].

In keeping with the philosophy that distribution functions are "the numbers of future" [18], we can look upon the lower distribution $F_{u v}$ as a measure of the chaos generated by $g$. We can also employ various common numerical quantities associated with this distribution. But for us the truly meaningful numerical measure is the area between the graphs of the distributions $F_{u v}^{*}$ and $F_{u v}$, namely the quantity

$$
\mu(g)=\left\|F_{u v}^{*}-F_{u v}\right\|=\int_{0}^{1}\left(F_{u v}^{*}-F_{u v}\right) d t=1-\int_{0}^{1} F_{u v} d t
$$

which, in this case, is equal to $0.7861936 \ldots$. This measure is particularly apt, since we shall show (see Theorems 2.1 and 2.2 below) that when $f$ is a map of zero topological entropy, then $F_{x y}^{*}=F_{x y}$ for all $x, y$ in $I$, whereas when $f$ has positive topological entropy, then there are always pairs of points $x, y$ in $I$ for which $F_{x y}<F_{x y}^{*}$.

The behavior of the tent map is particularly simple, but nevertheless illustrative. An arbitrary continuous map $f: I \rightarrow I$ may have a countably infinite system of maximal $\omega$-limit sets, each supporting positive topological entropy. However, the corresponding collection of minimal, mutually incomparable, lower distributions $F_{u v}$ is, surprisingly, always finite. And this result leads to a finite "spectral decomposition" of the map $f$ : the "subspaces" are the 
maximal $\omega$-limit sets; the "eigenvalues" are the corresponding minimal distributions $F_{u v}$ for which $F_{u v}^{*} \equiv 1$. As a measure of chaos, we may use either these eigenvalues or the largest of the numbers determined by them via (6).

Note further that if, for some given $f$ in $C(I, I)$ and some $x, y$ in $I$, there is an $\varepsilon>0$ such that $F_{x y}(\varepsilon)<1$, then the first relation in (1) holds; and similarly, if $F_{x y}^{*} \equiv 1$, then the second inequality in (1) holds. The converse implications are false. Thus, the conditions $F_{x y}<F_{x y}^{*} \equiv 1$ are refinements of the corresponding conditions in (1).

The remainder of this paper is organized as follows: The main results, along with some basic notions, several additional examples, and some remarks, are presented in $\S 2$; the most important of these is Theorem 2.4. Section 3 contains preliminary results on $\omega$-limit sets which extend some of Sharkovsky's results [16] (see Theorem 3.7). Section 5 is devoted to technical preliminaries on distance distribution functions on $\omega$-limit sets: here the basic results are Lemmas 5.2 and 5.5. Proofs of the main results are given in $\S 4$ and $\S 6$.

Throughout the paper, the term "topological entropy" is used only as a synonym for the existence of periodic points of certain periods: a map $f$ in $C(I, I)$ has zero topological entropy if and only if each of its periodic points has period $2^{n}$ for some nonnegative integer $n$ [10]. Other notions and terminology are introduced as needed in the text or may be found in the cited references, in particular [1].

\section{MAIN RESUlts}

For a given $f$ in $C(I, I)$, consider the system $\left\{\omega_{f}(x) ; x \in I\right\}$ of $\omega$-limit sets of $f$, partially ordered by inclusion. Following Sharkovsky, a maximal $\omega$-limit set of $f$ is of the second kind if it contains a periodic point and is infinite; otherwise it is a maximal $\omega$-limit set of $f$ of the first kind. The system of maximal $\omega$-limit sets of the second kind is always countable [15] (for more details see $\S 3$ ).

The dynamics is very complicated on maximal $\omega$-limit sets of the second kind. On the other hand, our initial result shows that it is very regular on sets of the first kind.

2.1 Theorem. Let $f \in C(I, I)$ and suppose that, for some $u, v$ in $I$, each of the sets $\omega_{f}(u)$ and $\omega_{f}(v)$ is contained in a maximal $\omega$-limit set of the first kind. Then

(i) $F_{u v}=F_{u v}^{*}$;

(ii) if, in addition, $\liminf _{i \rightarrow \infty} \delta_{u v}(i)=0$, then $F_{u v}=\chi_{(0, \infty)}$, i.e., $F_{u v}(t)=1$ for all $t>0$.

We say that an $\omega$-limit set $\omega$ of a map in $C(I, I)$ has a periodic decomposition of period $k$ if there is a compact periodic interval $J \subset I$ of period $k$ such that $\operatorname{Orb}(J) \supset \omega$, and such that for, $0 \leq i<k$, the convex hulls $\operatorname{conv}\left(\omega_{i}\right)$ of the set $\omega_{i}=f^{i}(J) \cap \omega$ are nonoverlapping, i.e., have at most endpoints in common. Any such set $\omega_{i}$ is called a periodic portion of $\omega$. (For more details see Lemma 3.2 and Theorem 3.7.)

The proof of Theorem 2.1, which is given in $\S 4$, is based on the fact (Theorem 3.6) that every infinite maximal $\omega$-limit set of the first kind has periodic decompositions of arbitrarily high periods. As an immediate consequence of Theorem 2.1 we have 
2.2 Theorem. Let $f$ in $C(I, I)$ have zero topological entropy. Then $F_{u v}=F_{u v}^{*}$ for all $u, v$ in I. If, in addition, $\liminf _{i \rightarrow \infty} \delta_{u v}(i)=0$ then $F_{u v}=\chi_{(0, \infty)}$.

Proof. If $f$ has zero topological entropy, then every maximal $\omega$-limit set is of the first kind ([15]; cf. also [4]), whence the result follows from Theorem 2.1. Alternatively, one may note that for a map of zero topological entropy every infinite $\omega$-limit set has periodic decompositions of period $2^{n}$ for every $n$ (cf., e.g. [19] or [4]) and apply Proposition 4.3.

This result shows that for a chaotic map of zero topological entropy, the distances between any two trajectories starting from a scrambled set are very small most of the time. This corresponds to the main result of [5].

Before stating the next result, which concerns the dynamics on maximal $\omega$ limit sets of the second kind, we recall that any such set $\omega$ has a maximal periodic decomposition, i.e., a periodic decomposition of maximal possible period, or equivalently, a decomposition such that the corresponding periodic portions are minimal, i.e., indecomposable (see $\S 3$ for more details).

2.3 Theorem. Let $f \in C(I, I)$ and let $\omega=\omega_{f}(x)$ be a maximal $\omega$-limit set of the second kind. Then there exists a nonempty perfect set $P \subset \omega$ and a probability distribution function $F$ such that $F(t)=0$ for $t<\varepsilon$, where $\varepsilon$ is a positive constant, and such that

(i) $F=F_{u v}<F_{u v}^{*}=\chi_{(0, \infty)}$ for any distinct $u, v$ in $P$;

(ii) if $S$ is a scrambled set for $f$ (or, more generally, if $\lim _{i n f} \operatorname{in}_{i \rightarrow \infty} \delta_{u v}(i)=0$ for any $u, v$ in $S$ ) such that $\omega_{f}(u) \subset \omega$ for any $u \in S$, then there are sets $S_{0}, S_{1}$ such that $S=S_{0} \cup S_{1}$ and $F_{u v} \geq F$ whenever $u, v \in S_{0}$, or $u, v \in S_{1}$;

(iii) if the minimal periodic portions of $\omega$ are pairwise disjoint, then $F_{u v} \geq F$ for any $u, v$ in $S$, i.e., $S_{1}=\varnothing$.

Note that, in the terminology of the theory of probabilistic metric spaces, the condition $F_{u v}=F$ may be interpreted as saying that, as measured by the lower distribution function $F_{u v}$, the points $u$ and $v$ are almost certainly discernible, whereas, in contrast, the condition $F_{u v}^{*}=\chi_{(0, \infty)}$ says that, as measured by $F_{u v}^{*}$, they are almost certainly indiscernible (see $[18, \S 12.10])$.

A combination of Theorems 2.1 and 2.3 yields our main result on the spectral decomposition of dynamical systems. The following terminology will simplify its formulation: We say that two points $u, v$ in $I$ are synchronous (with respect to a function $f$ in $C(I, I))$ if both $\omega_{f}(u)$ and $\omega_{f}(v)$ are contained in the same maximal $\omega$-limit set $\omega$, and if, for any periodic interval $J$ such that $\operatorname{Orb}(J) \supset \omega$, there is a $j \geq 0$ such that $f^{j}(u), f^{j}(v) \in J$. The spectrum of $f$, denoted by $\Sigma(f)$, is the set of minimal elements of the set $D(f)=\left\{F_{u v} ; u\right.$ and $v$ are synchronous $\}$. And the weak spectrum $\Sigma_{w}(f)$ of $f$ is the set of minimal elements of the set $D_{w}(f)=\left\{F_{u v} ; \liminf _{i \rightarrow \infty} \delta_{u v}(i)=0\right\}$. Any two elements of $\Sigma(f)$ (resp., $\Sigma_{w}(f)$ ) are incomparable; however, $\Sigma(f) \cup \Sigma_{w}(f)$ may contain comparable elements (see Remark 2.5). Nothing can be said about $\Sigma(f) \cap \Sigma_{w}(f)$; but we will show (see Remark 2.6) that $\Sigma(f) \subset D_{w}(f)$.

2.4 Theorem. Let $f \in C(I, I)$.

(A) If the topological entropy of $f$ is zero, then $\Sigma(f)=\Sigma_{w}(f)=\left\{\chi_{(0, \infty)}\right\}$.

(B) If the topological entropy of $f$ is positive, then the following hold:

(B1) Both the spectrum $\Sigma(f)$ and the weak spectrum $\Sigma_{w}(f)$ are finite (and nonempty). Specifically, $\Sigma(f)=\left\{F_{1}, \ldots, F_{m}\right\}$ for some $m \geq 1$, and 
$\Sigma_{w}(f) \backslash \Sigma(f)=\left\{F_{m+1}, \ldots, F_{n}\right\}$ where $n \geq m$. Furthermore, for each $i$ there is an $\varepsilon_{i}>0$ such that $F_{i}\left(\varepsilon_{i}\right)=0$.

For any positive integer $k \leq n$, let $\pi_{k}$ be the system of sets $P$ such that $\# P \geq 2$ and, for any distinct $u, v$ in $P, F_{k}=F_{u v}<F_{u v}^{*}=\chi_{(0, \infty)}$.

(B2) If $k \leq m$, then $\pi_{k}$ contains a nonempty perfect set $P_{k}$.

(B3) If, on the other hand, $m<k \leq n$, then $\pi_{k}$ is nonempty and any $P$ in $\pi_{k}$ is a two-point set.

(B4) If $S$ is a scrambled set for $f$ (or, more generally, if $\lim \inf _{i \rightarrow \infty} \delta_{u v}(i)$ $=0$ for any $u, v$ in $S$ ), then there are integers $i, j \leq m$ and a corresponding decomposition $S=S_{i} \cup S_{j}$ such that $F_{u v} \geq F_{i}$ if $u, v \in S_{i}$ and $F_{u v} \geq F_{j}$ if $u, v \in S_{j}$.

The proofs of Theorems 2.3 and 2.4 are given in $\S 6$.

2.5 Remark. For the tent map $g$, we have $\Sigma(g)=\Sigma_{w}(g)=\{F\}$, where $F$ is given by (5). More generally, consider the family of those trapezoids $g_{\lambda}=\min (g, \lambda)$ which have positive topological entropy, i.e., for which $\lambda \in$ $(0.8249080 \ldots, 1)[11]$. Each such $g_{\lambda}$ has a unique infinite maximal $\omega$-limit set, which is nowhere dense in $I$, and there is a distribution function $F_{\lambda}$ such that $\Sigma\left(g_{\lambda}\right)=\Sigma_{w}\left(g_{\lambda}\right)=\left\{F_{\lambda}\right\}$. It therefore follows from (B2) that there is a perfect set $P_{\lambda}$ such that, for any distinct $u, v$ in $P_{\lambda}$, we have $F_{\lambda}=F_{u v}<$ $F_{u v}^{*}=\chi_{(0, \infty)}$. Lastly, it can be shown that as $\lambda \nearrow 1, F_{\lambda} \searrow F$.

To exhibit an example illustrating (B3), we can consider the map $f$ in $C(I, I)$ which satisfies $f(0)=\frac{1}{2}, f\left(\frac{1}{4}\right)=1, f\left(\frac{3}{4}\right)=0$ and $f(1)=\frac{1}{2}$, and which is linear on the intermediate intervals. Clearly, $f$ is topologically transitive and hence has the unique maximal $\omega$-limit set $\omega=I$. This set has a decomposition into two periodic portions (of period 2 ), namely $\omega_{0}=\left[0, \frac{1}{2}\right]$ and $\omega_{1}=\left[\frac{1}{2}, 1\right]$. These have the fixed point $\frac{1}{2}$ in common and are interchanged by $f$. It is easy to verify that $f$ and the tent map $g$ have the same minimal lower distribution, namely the function $F$ given by (5), and that $F$ cannot be generated by synchronous points $u, v$ belonging only to one of the sets $\omega_{0}, \omega_{1}$. Thus, $\Sigma_{w}(f)=\{F\}$ and $F \notin \Sigma(f)$. Now it is readily seen that $\Sigma(f)=\left\{F_{1}\right\}$ where $F_{1}(t)=F(2 t)$ for any $t$. Thus, using the terminology of Theorem 2.4, we have $m=1$ and $n=2$. Similar ideas can be used to construct examples for any $m \geq 1$ and any $n \geq m$. Moreover, if $f$ has only $k$ different maximal $\omega$-limit sets of the second kind then, by Theorems 2.3 and 2.4, $m=\# \Sigma(f) \leq k$; and similarly (see the proof of Theorem 2.4), $n-m=\#\left(\Sigma_{w}(f) \backslash \Sigma(f)\right) \leq k$. In particular, if $f$ is topologically transitive then $\# \Sigma(f)=1$ and $\# \Sigma_{w}(f)=1$.

2.6 Remark. Conditions (B1) and (B2) of Theorem 2.4 lead to the following notion: An infinite set $S \subset I$ is said to be a strongly scrambled set for $f \in$ $C(I, I)$ if there is a distribution $F$ in $\Sigma(f)$ such that

$$
F_{x y}=F \quad \text { and } F_{x y}^{*}=\chi_{(0, \infty)},
$$

for any distinct points $x, y$ in $S$. Accordingly we say that $f$ is strongly chaotic or exhibits strong chaos. Clearly any strongly scrambled set is a scrambled set. However, since a map of zero topological entropy can have a scrambled set [19], Theorem 2.2, shows that the converse is false.

By definition, $\Sigma(f)$ is generated by pairs of synchronous points. It follows from Theorem 3.7 and Lemmas 5.2 and 5.3 that any $F$ in $\Sigma(f)$ may also be 
generated by a pair of synchronous points $u, v$ satisfying $\liminf _{i \rightarrow \infty} \delta_{u v}(i)=0$ or even $F_{u v}^{*}=\chi_{(0, \infty)}$ (see also the proof of Theorem 2.4 in $\left.\S 6\right)$. This implies that the restriction $F_{u v}^{*}=\chi_{(0, \infty)}$ is inessential, and further that $\Sigma(f) \subset D_{w}(f)$.

2.7 Remark. In the theory of dynamical systems the term "spectral decomposition" usually refers to a decomposition of the set of $\omega$-limit points into a (generally infinite) collection of maximal $\omega$-limit sets or, e.g., to a similar decomposition of the set of nonwandering points (see, e.g., [12] or [14]). For us, such a decomposition is only a basis for determining the corresponding minimal lower distributions. By omitting those $\omega$-limit sets which lead to distributions that are "negligible" in the sense that they are comparable with a minimal one, we arrive at a finite collection of distribution functions-our spectrum.

2.8 Remark. Although the details remain to be worked out, it is virtually certain that strong chaos is stable in the following sense: If $f$ has a strongly scrambled set $P$ with corresponding lower distribution function $F$, and if $g$ is (uniformly) sufficiently close to $f$, then $g$ has a strongly scrambled set and a corresponding minimal lower distribution $G$ such that the difference between $G$ and $\min (F, G)$ is small. However, the difference between $F$ and $G$ may be large, with $F>G$. For example, we may take $f$ to be topologically transitive in a small interval $J \subset I$, let $f(x)=x$ in $I \backslash J$, and let $g$ be a perturbation of $f$ that is topologically transitive in $I$. In other words, the distribution $G$ satisfies a lower semicontinuity condition. Note that, contrary to this, chaos in the sense of $\mathrm{Li}$ and Yorke is not stable [13].

There are interesting connections between points of discontinuity of the minimal distributions in $\Sigma(f) \cup \Sigma_{w}(f)$ and the periodic orbit structure of $f$ which remain to be explored. For example, it appears that if $t$ is a point of discontinuity of such a distribution, then there are periodic points $p$ and $q$ in $I$ such that $|p-q|=t$.

\section{BASIC PROPERTIES OF MAXIMAL $\omega$-LIMIT SETS}

Our main aim in this section is to prove Theorems 3.6 and 3.7. We begin with the following description of $\omega$-limit sets which is due to Sharkovsky [15].

3.1 Theorem. Let $f \in C(I, I)$ and let $x, y \in I$. Then

(i) if $a \in \omega_{f}(x) \cap \omega_{f}(y)$ is a limit point from the left (resp., from the right) of both $\omega_{f}(x)$ and $\omega_{f}(y)$, then $\omega_{f}(x) \cup \omega_{f}(y)$ is an $\omega$-limit set of $f$;

(ii) the system $A_{2}(f)$ of maximal $\omega$-limit sets of the second kind is countable;

(iii) if $\omega \in A_{2}(f)$, then $\omega$ is perfect;

(iv) if $\omega \in A_{2}(f)$ and if $\omega_{f}(x) \subset \omega$, then $f^{i}(x) \in \omega$ for all sufficiently large $i$;

(v) if $\omega \in A_{2}(f)$ and if $\omega_{f}(x) \subset \omega$, then the set $\left\{v \in \omega ; \omega_{f}(v)=\omega_{f}(x)\right\}$ is dense in $\omega$.

The next result must be known but we are not able to give a reference.

3.2 Lemma. Let $f \in C(I, I)$, and let $\omega=\omega_{f}(x)$ be an infinite $\omega$-limit set which has a proper periodic portion, i.e., assume that there is a compact periodic interval $J$ of period $m$ such that $\omega \backslash J \neq \varnothing \neq \omega \cap \operatorname{int}(J)$. Then $\omega$ has a periodic decomposition of period $n$, for some $n$ with $1<n \leq m$.

Proof. Let $K$ be a minimal interval of the form $K=f^{n(1)}(J) \cap \cdots \cap f^{n(s)}(J)$, for some $s \geq 1$, such that $\omega \cap \operatorname{int}(K) \neq \varnothing$. Then $f^{m}(K) \subset K$. Let $n>0$ be 
the minimal integer such that $f^{n}(K) \subset K$. Since $\omega \cap \operatorname{int}(K) \neq \varnothing, f^{i}(x) \in K$, for some $i$ (and hence, $\omega \subset \operatorname{Orb}(K))$. Let $g=f^{n}$, and let $\omega_{j}=\omega_{g}\left(f^{i+j}(x)\right)$, for $0 \leq j<n$. We claim that the sets $\omega_{j}$ form a periodic decomposition of $\omega$.

It is easy to see that $\omega_{0} \cup \cdots \cup \omega_{n-1}=\omega$ and that $f\left(\omega_{j}\right)=\omega_{j+1(\bmod n)}$, for any $j$. We have $\omega_{0} \subset K$. If $\omega_{k} \cap \operatorname{int}(K) \neq \varnothing$ for some $0<k<n$, then we get easily $\omega_{k} \subset K$, and $\omega_{0}=f^{n-k}\left(\omega_{k}\right) \subset f^{n(1)+n-k}(J) \cap \cdots \cap f^{n(s)+n-k}(J)=L$. But $K$ is minimal, hence $L \supset K$. On the other hand, $f^{k}(L) \subset K$, hence $f^{k}(K) \subset K$ which is impossible since $n>k$ is minimal. Thus $\omega_{k} \cap \operatorname{int}(K)=\varnothing$ and similarly we can show that $\omega_{k} \cap \operatorname{int}\left(f^{j}(K)\right)=\varnothing$ for any $j \neq k(\bmod n)$. To complete the proof note that $n>1$, since otherwise $\omega \subset K \subset J$, and that $K$ contains a compact subinterval $K_{0}$ with $\omega_{0} \subset K_{0}=f^{n}\left(K_{0}\right)$, and hence, $\omega_{j} \subset f^{j}\left(K_{0}\right)$ for any $j$.

3.3 Lemma. Let $f \in C(I, I)$, let $\omega$ be an $\omega$-limit set with no proper periodic portions, and let $U=f(U)$ be the minimal compact invariant interval containing $\omega$. If $J, K$ are compact intervals such that $K \subset \operatorname{int}(U)$ and $J \cap \omega$ is uncountable then $f^{i}(J) \supset K$ for all sufficiently large $i$.

Proof. We may assume that $J \subset U$. Recall that any interval $G$ such that $G \cap \omega_{f}(y)$ is infinite for some $y$ contains a periodic point [6]; see also [19]. It follows that $J$ contains periodic points $p<q$ such that $[p, q] \cap \omega$ is infinite. Consequently, if $m>0$ is a common multiple of the periods of $p$ and $q$, then $f^{m}([p, q]) \supset[p, q]$, and $L=\bigcup_{i=1}^{\infty} f^{i m}([p, q])$ is a periodic interval with $f^{m}(L)=L$. Moreover, $L \cap \omega$ is infinite and hence $\omega \subset \bar{L}$, where $\bar{L}$ is the closure of $L$, since $\omega$ has no proper periodic portions. Then $\omega \subset \bigcap_{i=1}^{m} f^{i}(\bar{L})=$ $M$, and $M \supset f(M) \supset \omega$, whence $U \subset M$ because of the minimality of $U$. Lastly, it is easy to see that $f^{j}([p, q]) \supset K$ for sufficiently large $j$.

3.4 Lemma. Let $f \in C(I, I)$ and let $u$, $v$ be in $I$. Let $\left\{U_{i}\right\}_{i=1}^{\infty}$ and $\left\{V_{i}\right\}_{i=1}^{\infty}$ be compact intervals with $\lim _{i \rightarrow \infty} U_{i}=u$ and $\lim _{i \rightarrow \infty} V_{i}=v$, and such that for any $i$ and $j$ there are positive integers $u(i, j)$ and $v(i, j)$ such that $f^{u(i, j)}\left(U_{i}\right) \supset V_{j}$ and $f^{v(i, j)}\left(V_{i}\right) \supset U_{j}$. Then $\{u, v\} \subset \omega_{f}(y)$, for some $y$ in $I$.

Proof. Define a decreasing sequence $\left\{J_{i}\right\}_{i=1}^{\infty}$ of compact intervals and an increasing sequence $\{n(i)\}_{i=1}^{\infty}$ of positive integers as follows: $J_{1}=U_{1}$ and $n(1)=u(1,2)$. Then $f^{n(1)}\left(J_{1}\right) \supset V_{2}$; choose $J_{2} \subset J_{1}$ such that $f^{n(1)}\left(J_{2}\right)=V_{2}$. Take $n(2)=n(1)+v(2,3)$. Then $f^{n(2)}\left(J_{2}\right)=f^{v(2,3)}\left(V_{2}\right) \supset U_{3}$ and there is a $J_{3} \subset J_{2}$ such that $f^{n(2)}\left(J_{3}\right)=U_{3}$. Then there are $J_{4}$ and $n(3)$ such that $f^{n(3)}\left(J_{4}\right)=V_{4}$, etc. Let $y \in \bigcap_{i=1}^{\infty} J_{i}$. Since the trajectory of $y$ visits every neighborhood of $u$ and every neighborhood of $v$, the result follows.

3.5 Lemma. Let $f \in C(I, I)$ and let $\omega=\omega_{f}(x)$ be a maximal $\omega$-limit set with no proper periodic portions. If $J$ is an interval such that $J \cap \omega$ is uncountable then $J \cap \omega$ contains a periodic point.

Proof. Let $U$ be as in Lemma 3.3. We may assume that $J=[a, b] \subset \operatorname{int}(U)$. By Lemma 3.3, $J \subset f^{k}(J)$, for some $k>0$, hence $J$ contains a periodic point $p=f^{k}(p)$. One of the sets $[a, p] \cap \omega,[p, b] \cap \omega$, say $[a, p] \cap \omega$, is uncountable. Again by Lemma 3.3, we have $[a, p] \subset \operatorname{int}\left(f^{k s}([a, p])\right.$ for some $s>0$. Hence there is a point $q$ such that $f^{k s}(q)<a<q<p$. Let $f^{k s}(q)=u_{1}$ 
and $q=u_{2}$. Then $f^{k s}\left(\left[u_{2}, p\right]\right) \supset\left[u_{1}, p\right]$, and since $f^{k s}$ is continuous, there is a sequence $u_{1}<u_{2}<\cdots<p$ such that $f^{k s}\left(u_{j+1}\right)=u_{j}$, for any $j$. Let $u=\lim _{j \rightarrow \infty} u_{j}$, and $U_{j}=\left[u_{j}, u\right]$.

Let $v \in \omega \cap U_{1}$ be such that $\omega_{f}(v)$ is infinite and $G \cap \omega$ is uncountable for any neighborhood $G$ of $v$. Note that such a $v$ always exists, since if $\omega$ is a maximal $\omega$-limit set of the first kind then any $\omega_{f}(z)$ with $z \in \omega$ is infinite (otherwise $\omega_{f}(z)$ would be a periodic orbit) and if $\omega$ is of the second kind, then by $(\mathrm{v})$ of Theorem 3.1, there is even a $v$ such that $\omega_{f}(v)=\omega$.

Let $\left\{V_{i}\right\}_{i=1}^{\infty}$ be neighborhoods of $v$ with $\lim _{i \rightarrow \infty} V_{i}=v$. By Lemmas 3.3 and 3.4 , there is a $y$ such that $\{u, v\} \subset \omega_{f}(y)$. Since $v \in \omega_{f}(x)$, we have $\omega_{f}(v) \subset \omega_{f}(x) \cap \omega_{f}(y)$, and since $\omega_{f}(v)$ is infinite, by (i) of Theorem 3.1, $\omega_{f}(x) \cup \omega_{f}(y)$ is an $\omega$-limit set. But $\omega_{f}(x)$ is maximal, hence $\omega_{f}(y) \subset \omega_{f}(x)$, and hence, $u \in \omega_{f}(x)$. Note that $u=f^{k s}(u) \in J$.

The next result is basic for the proof of Theorem 2.1 .

3.6 Theorem. Let $f \in C(I, I)$ and let $\omega=\omega_{f}(x)$ be an infinite maximal $\omega$-limit set of $f$. Then $\omega$ is of the first kind if and only if $\omega$ has periodic decompositions of arbitrarily high periods.

Proof. One implication is immediate. For if $\omega$ has periodic decompositions of arbitrarily high periods then clearly $\omega$ contains no periodic points. To prove the reverse implication assume that $\omega$ is a maximal $\omega$-limit set and that $\omega_{1}$ is its minimal periodic portion, of maximal possible period $m \geq 1$. Let $g=f^{m}$. It is easy to see that $\omega_{1}$ is a maximal $\omega$-limit set of $g$ (we have $\omega_{1}=\omega_{g}\left(f^{i}(x)\right.$ ) for some $i<m$ ) and that $\omega_{1}$ is indecomposable. But then $\omega_{1}$ contains a periodic point. To see this apply Lemma 3.5 if $\omega_{1}$ is uncountable, and use the fact that any countable $\omega$-limit set contains a periodic point [17] otherwise. (Actually, the infinite maximal $\omega$-limit sets are always uncountable.) Thus, $\omega$ is of the second kind.

The following theorem summarizes the above results.

3.7 Theorem. Let $f \in C(I, I)$ and let $A_{1}(f), A_{2}(f)$ be the classes of infinite maximal $\omega$-limit sets of $f$ of the first and second kind, respectively. Let $\omega_{f}(x)$ be an infinite $\omega$-limit set.

(i) If $\omega_{f}(x)$ has no minimal periodic portions, then $\omega_{f}(x)$ has periodic decompositions of arbitrarily high periods.

(ii) If $\omega_{f}(x)$ has a minimal periodic portion (of period $m \geq 1$ ), then $\omega_{f}(x) \subset$ $\omega$, for some $\omega \in A_{2}(f)$.

(iii) If $\omega_{1} \in A_{1}(f)$ and $\omega_{1} \neq \omega$ for some $\omega \in A_{1}(f) \cup A_{2}(f)$, then $\omega_{1} \cap \omega=$ $\varnothing$. In particular, two distinct periodic portions of sets in $A_{1}(f)$ with the same period have no common points.

(iv) The intersection of any two distinct sets in $A_{2}(f)$ is finite and, if nonempty, is the orbit of a periodic point. More precisely, if $\omega_{1}$ and $\omega_{2}$ are distinct minimal periodic portions of any two sets in $A_{2}(f)$, and if $U=\operatorname{conv}\left(\omega_{1}\right)$ and $V=$ $\operatorname{conv}\left(\omega_{2}\right)$, then $U \cap V=\varnothing$, or $U$ and $V$ have just one point in common, or $U \subset \operatorname{int}(V)$, or $V \subset \operatorname{int}(U)$.

(v) If $\omega \in A_{2}(f)$ then the set $\omega \cap \operatorname{Per}(f)$, where $\operatorname{Per}(f)$ is the set of periodic points of $f$, is dense in $\omega$.

(vi) Let $\omega \in A_{2}(f)$. Then there is a minimal compact periodic interval $U$ (of period $m \geq 1$ ) such that $\operatorname{Orb}(U) \supset \omega$. Moreover, if $J, K$ are compact 
intervals such that $J \cap \omega$ is infinite and $K \subset \operatorname{int}(U)$, then $f^{i+j m}(J) \supset K$ for some $i$ and any sufficiently large $j$. We will describe this situation by saying that $f \mid \omega$ is strongly transitive in $U$.

Proof. (i), (ii), (v) and (vi) are easy consequences of Lemma 3.2, Theorem 3.6, Lemma 3.5, and Lemma 3.3 and Theorem 3.6, respectively.

(iii) Assume that $P=\omega_{1} \cap \omega \neq \varnothing$. If $P$ is finite, then $P$ contains a periodic point since $f(P) \subset P$, and this is impossible. If $P$ is infinite, then (i) of Theorem 3.1 implies that $\omega_{1}=\omega$.

(iv) It is easy to see that $\omega_{1}, \omega_{2} \in A_{2}\left(f^{m}\right)$, for some $m$, hence we may assume that $m=1$. Thus if $U \cap V=J$ is an interval and neither $U \subset V$ nor $V \subset U$, then $J \cap \omega_{1}$ and $J \cap \omega_{2}$ are uncountable (Theorem 3.1). Let $u \in \omega_{1}$ and $v \in \omega_{2}$ be points interior to $J$ such that $\omega_{f}(u)=\omega_{1}$ and $\omega_{f}(v)=\omega_{2}$ (Theorem 3.1). By Lemmas 3.3 and 3.4, $u, v \in \omega_{f}(z)$, for some $z$, and since $\omega_{1}, \omega_{2}$ are maximal, we have $\omega_{1}=\omega_{2}$-a contradiction.

It remains to show that $U$ and $V$ cannot have a common endpoint if $U \subset$ $V$, but this follows by (i) and (iii) of Theorem 3.1.

\section{Proof of Theorem 2.1}

4.1 Lemma. Let $f \in C(I, I)$. Then, for any $t, \lambda$ in $(0,1)$ there is anteger $n(t, \lambda)$ with the following property: If $A$ is a periodic set of period $m \geq n(t, \lambda)$, and if the convex hulls of the sets $f^{s}(A)$, for $s<m$, are nonoverlapping, then for any $u, v$ in $A, F_{u v}(t)>\lambda$.

Proof. Fix $t$ and $\lambda$. Let $n(t, \lambda)$ be such that $(n(t, \lambda)-1 / t) / n(t, \lambda)>\lambda$. Since there are at most $1 / t$ distinct sets $f^{s}(A)$ with $\operatorname{diam} f^{s}(A) \geq t$, we have $F_{u v}(t) \geq \frac{1}{m} \#\left\{s<m ; \operatorname{diam} f^{s}(A)<t\right\} \geq(m-1 / t) / m>\lambda$.

4.2 Lemma. Let $f \in C(I, I)$, and let $\omega_{f}(x)$ have periodic portions of arbitrarily high periods. Then for any $\varepsilon>0$ there is a periodic point $p$ such that $F_{x p}(t)>1-\varepsilon$ for $t \geq \varepsilon$.

Proof. Let $J$ be a compact periodic interval of period $m \geq n(\varepsilon, 1-\varepsilon$ ) (cf. Lemma 4.1) with $\operatorname{Orb}(J) \supset \omega_{f}(x)$. Clearly $f^{i}(x) \in J$ for some $i$, and since $f^{m}(J)=J$, there is a periodic point $q$ in $J$. Let $p \in \operatorname{Orb}(q)$ be such that $f^{i}(p)=q$. Now apply Lemma 4.1.

4.3 Proposition. Let $f \in C(I, I)$, and suppose that each of $\omega_{f}(u), \omega_{f}(v)$ is either finite or decomposable into an arbitrarily high number of periodic portions. Then $F_{u v}=F_{u v}^{*}$.

Proof. Let $\varepsilon>0$, and let $p, q$ be periodic points such that $F_{u p}(t)>1-\varepsilon$ and $F_{v q}(t)>1-\varepsilon$ if $t \geq \varepsilon$. Such a $p$ exists trivially if $\omega_{f}(u)$ is finite (and hence a cycle), and otherwise it exists by Lemma 4.2 ; and similarly for $q$. Then $\#\left\{i<k ; \delta_{u p}(i) \geq \varepsilon\right\}<\varepsilon k$ and $\#\left\{i<k ; \delta_{v q}(i) \geq \varepsilon\right\}<\varepsilon k$ whenever $k$ is sufficiently large. For any such $k$,

$$
\frac{1}{k} \xi(p, q, k, t-2 \varepsilon)-2 \varepsilon<\frac{1}{k} \xi(u, v, k, t)<\frac{1}{k} \xi(p, q, t+2 \varepsilon)+2 \varepsilon
$$

( $\xi$ is defined by (2)), and for $k \rightarrow \infty$,

$$
F_{p q}(t-2 \varepsilon)-2 \varepsilon \leq F_{u v}(t) \leq F_{u v}^{*}(t) \leq F_{p q}(t+2 \varepsilon)+2 \varepsilon
$$


and a simple calculation gives $\left\|F_{u v}^{*}-F_{u v}\right\| \leq 8 \varepsilon$. Equivalently, we have $L\left(F_{u v}, F_{u v}^{*}\right) \leq L\left(F_{u v}, F_{p q}\right)+L\left(F_{p q}, F_{u v}^{*}\right) \leq 2 \varepsilon+2 \varepsilon=4 \varepsilon$ where $L$ is the Lévy metric [18]. Since $\varepsilon$ is arbitrary, the result follows.

4.4 Proof of Theorem 2.1. The first part follows by Theorem 3.6 and Proposition 4.3. So let $\liminf _{i \rightarrow \infty} \delta_{u v}(i)=0$. Then the maximal $\omega$-limit sets $\omega_{u}, \omega_{v}$, containing $\omega_{f}(u)$ and $\omega_{f}(v)$, respectively, have a nonempty intersection. If $\omega_{u}$ is finite, i.e., if it is the orbit of a periodic point, then trivially $\omega_{u}=\omega_{v}$ and $\lim _{i \rightarrow \infty} \delta_{u v}(i)=0$, which implies (ii). So assume that $\omega_{u}, \omega_{v}$ are infinite. Then by (iii) of Theorem 3.7, $\omega_{u}=\omega_{v}=\omega$. Let $J$ be a compact periodic interval with $\operatorname{Orb}(J) \supset \omega$. Clearly $f^{i}(u), f^{i}(v)$ are in $J$, for some $i$, and hence $f^{i+j}(u), f^{i+j}(v) \in f^{j}(J)$, for any $j$. Since the period of $J$ can be arbitrarily high, Lemma 4.1 implies the result.

\section{Probability distribution functions on maximal $\omega$-Limit SETS}

Our main results in this section are Lemmas 5.2 and 5.5.

5.1 Lemma. Let $f \in C(I, I)$ and let both $F_{x y}^{*}$ and $F_{x y}$ be continuous at $t$. Then, for any $\varepsilon>0$, there are positive integers $k, q$, arbitrarily large, and $\delta>0$ such that

$$
\frac{1}{k} \xi(u, v, k, t)<F_{x y}(t)+\varepsilon
$$

and

$$
\frac{1}{q} \xi(u, v, q, t)>F_{x y}^{*}(t)-\varepsilon
$$

whenever $|u-x|<\delta$ and $|v-y|<\delta$. (Here $\xi$ is the function defined in (2).) Proof. Choose $\varepsilon_{1}>0$ such that $F_{x y}\left(t+2 \varepsilon_{1}\right)<F_{x y}(t)+\varepsilon / 2$ and $F_{x y}^{*}\left(t-2 \varepsilon_{1}\right)>$ $F_{x y}^{*}(t)-\varepsilon / 2$. Then choose $k$ such that $\frac{1}{k} \xi\left(x, y, k, t+2 \varepsilon_{1}\right)<F_{x y}\left(t+2 \varepsilon_{1}\right)+\varepsilon / 2$. The first inequality follows from the fact that $\xi(u, v, k, t)<\xi\left(x, y, k, t+2 \varepsilon_{1}\right)$ whenever $\delta>0$ is sufficiently small. The argument for the second inequality is similar.

5.2 Lemma. Let $f \in C(I, I)$, and let $\omega_{1}$ and $\omega_{2}$ be maximal $\omega$-limit sets of the second kind. Assume that there are periodic intervals $U, V$ and a countable set $Q \subset I^{2}$ of pairs $(u, v)$ such that

$$
f \mid \omega_{1} \text { is strongly transitive in } \operatorname{int}(U) \text { and } f \mid \omega_{2} \text { in } \operatorname{int}(V)
$$

and, furthermore, that

$$
u \in \omega_{1} \cap \operatorname{int}(U) \text { and } v \in \omega_{2} \cap \operatorname{int}(V) \quad \text { if }(u, v) \in Q .
$$

Then there are points $x \in \omega_{1} \cap U$ and $y \in \omega_{2} \cap V$ such that, for any $t>0$,

$$
F_{x y}(t) \leq \inf \left\{F_{u v}(t) ;(u, v) \in Q\right\}
$$

and

$$
F_{x y}^{*}(t) \geq \sup \left\{F_{u v}^{*}(t) ;(u, v) \in Q\right\}
$$

Proof. Let $T$ be a countable set, dense in $I$, and such that, for any $(u, v) \in Q$ and any $t \in T$, both $F_{u v}$ and $F_{u v}^{*}$ are continuous at $t$. Let $\left\{t_{j}\right\}_{j=1}^{\infty}$ and $\{u(j), v(j)\}_{j=1}^{\infty}$ be sequences of points from $T$ and $Q$, respectively, such that 
for any $t \in T$ and any $(u, v) \in Q, t=t_{j}, u=u(j)$ and $v=v(j)$ for infinitely many $j$.

Next, using induction, we define positive integers

$$
k(1)<q(1)<k(2)<q(2)<\cdots<k(i)<q(i)<\cdots
$$

and decreasing sequences $\left\{U_{i}\right\}_{i=1}^{\infty}$ and $\left\{V_{i}\right\}_{i=1}^{\infty}$ of compact intervals with

$$
\lim _{i \rightarrow \infty} \operatorname{diam}\left(U_{i}\right)=\lim _{i \rightarrow \infty} \operatorname{diam}\left(V_{i}\right)=0,
$$

and such that for any $u \in U_{n}$ and $v \in V_{n}$ and any $j \leq n$,

$$
\frac{1}{k(j)} \xi\left(u, v, k(j), t_{j}\right) \leq F_{u(j) v(j)}\left(t_{j}\right)+\frac{1}{j}
$$

and

$$
\frac{1}{q(j)} \xi\left(u, v, q(j), t_{j}\right) \geq F_{u(j) v(j)}^{*}\left(t_{j}\right)-\frac{1}{j} .
$$

To do this, we take $U_{1}=U, V_{1}=V, k(1)=1, q(1)=2$, and assume that $U_{n}, V_{n}, k(n)$ and $q(n)$ have been defined such that $f^{j}\left(U_{n}\right) \cap \omega_{1}$ and $f^{j}\left(V_{n}\right) \cap \omega_{2}$ are infinite whenever $j$ is sufficiently large. Since $U$ and $V$ are periodic, by (8) and (9) there is some $s>q(n)$ such that $u(n+1) \in f^{s}\left(U_{n}\right)$ and $v(n+1) \in f^{s}\left(V_{n}\right)$. Let $a \in U_{n}$ and $b \in V_{n}$ be such that $f^{s}(a)=u(n+1)$ and $f^{s}(b)=v(n+1)$. Then clearly $F_{a b}=F_{u(n+1) v(n+1)}$ and $F_{a b}^{*}=F_{u(n+1) v(n+1)}^{*}$. Now the existence of $U_{n+1} \subset U_{n}, V_{n+1} \subset V_{n}, \dot{k}(n+1)$ and $q(n+1)$ follows easily by Lemma 5.1. (We take as $U_{n+1}$ and $V_{n+1}$ compact neighborhoods of $a$ and $b$, respectively, with $\operatorname{diam}\left(U_{n}\right)>2 \operatorname{diam}\left(U_{n+1}\right)$ and $\operatorname{diam}\left(V_{n}\right)>2 \operatorname{diam}\left(V_{n+1}\right)$. It is easy to see (cf. (iii) of Theorem 3.1) that $a, b, U_{n+1}$ and $V_{n+1}$ can be chosen such that both $f^{s}\left(U_{n+1}\right) \cap \omega_{1}$ and $f^{s}\left(V_{n+1}\right) \cap \omega_{2}$ are infinite.)

Take $x \in \bigcap_{j=1}^{\infty} U_{j}$ and $y \in \bigcap_{j=1}^{\infty} V_{j}$. For any $t \in T$ and any $(u, v)$ in $Q$, take $j$ such that $t=t_{j}, u=u(j)$ and $v=v(j)$. Since $x \in U_{j}$ and $y \in V_{j}$, (12) applies with $u=x$ and $v=y$. Since $j$ can be arbitrarily large we have $F_{x y}(t) \leq F_{u v}(t)$. This implies (10) for any $t \in T$, and since $T$ is dense in $I$, for any $t$. The argument for (11) is similar.

We already have $x \in U=U_{1}$ and $y \in V$. It remains to show that $x$ can be chosen in $\omega_{1}$ and $y$ in $\omega_{2}$. Let $w \in \omega_{1} \cap U$ be such that $\omega_{f}(w)=\omega_{1}$, and let $\left\{W_{i}\right\}_{i=1}^{\infty}$ be a decreasing sequence of compact neighborhoods of $w$ with $\lim _{i \rightarrow \infty} W_{i}=w$. Now apply Lemma 3.4 with $u$ replaced by $x, v$ by $w$ and $V_{i}$ by $W_{i}$. Since $\omega_{1}$ is maximal, we obtain $\omega_{f}(x) \subset \omega_{1}$. Similarly, we have that $\omega_{f}(y) \subset \omega_{2}$. Now by (iv) of Theorem 3.1, $f^{n}(x) \in \omega_{1}$ and $f^{n}(y) \in \omega_{2}$, for some $n$. It is easy to see that $n$ can be chosen such that $f^{n}(x) \in U$ and $f^{n}(y) \in V$. Replace $x$ by $f^{n}(x)$ and $y$ by $f^{n}(y)$. Clearly (10) and (11) remain valid.

5.3 Lemma. Let $f \in C(I, I)$, let $\omega_{1}, \omega_{2}$ be maximal $\omega$-limit sets of $f$ of the second kind, and let $U$ and $V$ be minimal compact periodic intervals with $\operatorname{Orb}(U) \supset \omega_{1}$ and $\operatorname{Orb}(V) \supset \omega_{2}$. Then for any $u \in U \cap \omega_{1}$ and $v \in V \cap \omega_{2}$ there are $u^{*} \in \operatorname{int}(U) \cap \omega_{1}$ and $v^{*} \in \operatorname{int}(V) \cap \omega_{2}$ such that $F_{u v}=F_{u^{*} v^{*}}$.

Proof. First we show that $F_{u v}=F_{u(0) v(0)}$ where $u(0) \in \omega_{1} \cap U$ and $v(0) \in$ $\omega_{2} \cap V$ are suitable nonperiodic points. To do this take $u(0)=u$ if $u$ is not periodic; otherwise by $(\mathrm{v})$ of Theorem 3.1 there is a nonperiodic point $u(0)$ in 
$U \cap \omega_{1}$ such that $\omega_{f}(u)=\omega_{f}(u(0))$, and one can easily verify that $u(0)$ can even be chosen such that $\lim _{i \rightarrow \infty} \delta_{u(0) u}(i)=0$. Then clearly $F_{u v}=F_{u(0) v}$. The point $v(0)$ is defined similarly.

Now let $m>0$ be a multiple of the periods of $U$ and $V$. Since $f^{m}\left(U \cap \omega_{1}\right)=U \cap \omega_{1}$, there is a sequence $\{u(i)\}_{i=0}^{\infty}$ of points in $U \cap \omega_{1}$ such that $f^{m}(u(i+1))=u(i)$ for any $i>0$. Choose $\{v(i)\}_{i=0}^{\infty}$ in $V \cap \omega_{2}$ similarly. Now the points $u(i), v(i)$ are not periodic, hence for some $j, u(j) \in \operatorname{int}(U) \cap \omega_{1}$ and $v(j) \in \operatorname{int}(V) \cap \omega_{2}$. Take $u^{*}=u(j)$ and $v^{*}=v(j)$.

In the proof of our basic Lemma 5.5 we use methods of symbolic dynamics as developed, e.g., in [3] (cf. also [19]). Denote by $X$ the space $\{0,1\}^{N}$ of sequences of zeros and ones, equipped with the metric of pointwise convergence (e.g., $\left.\rho(\{\alpha(i)\},\{\beta(i)\})=\sum_{i=1}^{\infty}|\alpha(i)-\beta(i)| 2^{-i}\right)$. The following result will simplify our argument.

5.4 Lemma. Let $\left\{N_{i}\right\}_{i=1}^{\infty}$ be a decomposition of the set $N$ of positive integers into infinite subsets. Then there is an uncountable Borel set $B \subset X$ such that, for any distinct $\alpha=\{\alpha(i)\}_{i=1}^{\infty}$ and $\beta=\{\beta(i)\}_{i=1}^{\infty}$ in $B$ and any $n$,

$\left\{j \in N_{n} ; \alpha(j) \neq \beta(j)\right\}$ is infinite.

Proof. Let $\xi$ be an irrational number. Define $\psi:[0,1] \rightarrow X$ as follows: $\psi(t)=\left\{t_{j}\right\}_{j=1}^{\infty}$ where $t_{i}=0$ if $E(t+i \xi) \in\left[0, \frac{1}{2}\right)$, and $t_{i}=1$ otherwise. Here $E(x) \in[0,1)$ denotes the fractional part of $x$. Since, for any $i, E(t+i \xi)$ has at most two points of discontinuity in $[0,1]$, the function

$\psi$ is continuous in $[0,1] \backslash D$, where $D$ is a countable set.

It is well known that the sequence $\{E(i \xi)\}_{i=1}^{\infty}$ is dense in $[0,1]$. It follows that if $\psi(s)=\left\{s_{i}\right\}_{i=1}^{\infty}$ and $\psi(t)=\left\{t_{i}\right\}_{i=1}^{\infty}$ then

$t_{i} \neq s_{i}$ for infinitely many $i$ if $t \neq s$.

Now, for any $n$, let $a_{n 1}<a_{n 2}<\cdots$ be the elements of $N_{n}$. Define a map $\pi_{n}: X \rightarrow X$ such that $\pi_{n}(\alpha)=\left\{\alpha\left(a_{n i}\right)\right\}_{i=1}^{\infty}$ for any $\alpha=\{\alpha(i)\}_{i=1}^{\infty}$ in $X$. It is easy to see that $\pi_{n}$ is a continuous map onto $X$. Let $A=\psi([0,1] \backslash D)$. By (15) and (16), $A$ is an uncountable Borel set, hence the same is true for $B_{n}=\pi_{n}^{-1}(A)$, for any $n$. Put $B=\bigcap_{n=1}^{\infty} B_{n}$. Clearly $B$ is a Borel set, and since $\pi_{n}(B)=\pi_{n}\left(B_{n}\right)=A, B$ is uncountable (note that the sets $N_{i}$ are pairwise disjoint). Condition (14) now follows from (16).

5.5 Lemma. Let $f \in C(I, I)$, and let $\omega=\omega_{f}(z)$ be a maximal $\omega$-limit set of the second kind. Let $U$ be a minimal compact periodic interval with $\operatorname{Orb}(U) \supset \omega$, and let $x_{0}, x_{1}$ be in $U \cap \omega$. Then there is a nonempty perfect set $P \subset \omega$ such that, for any distinct $u, v$ in $P$,

$$
F_{u v} \leq F_{x_{0} x_{1}} \text { and } F_{u v}^{*} \geq F_{x_{0} x_{1}}^{*} .
$$

Proof. We use ideas from Lemma 5.2, combined with methods of symbolic dynamics. Let $T$ be a countable subset of $I$, dense in $I$ and such that both $F_{x_{0} x_{1}}$ and $F_{x_{0} x_{1}}^{*}$ are continuous at each $t \in T$, and let $\left\{t_{j}\right\}_{j=1}^{\infty}$ be a sequence of points from $T$ that contains every $t$ from $T$ infinitely many times. Let $X_{n}=\{0,1\}^{n}$, for $n=1,2, \ldots$, and define a system of compact intervals $\left\{I_{\alpha} ; \alpha \in X_{j}\right\}_{j=1}^{\infty}$ and positive integers

$$
k(1)<q(1)<k(2)<q(2)<\cdots<k(j)<q(j)<\cdots
$$


such that, for every $\alpha=\alpha(1) \alpha(2) \cdots \alpha(n)$ and $\beta=\beta(1) \beta(2) \cdots \beta(n)$ in $X_{n}$, the following is true:

$$
\begin{gathered}
f^{j}\left(I_{\alpha}\right) \cap \omega \text { is infinite if } j>k(n+1) ; \\
\text { if } \alpha \neq \beta \text { then } I_{\alpha} \cap I_{\beta}=\varnothing ;
\end{gathered}
$$

if $\gamma$ is in $X_{k}$ for some $k$ then $I_{\alpha \gamma} \subset I_{\alpha} \subset \operatorname{int}(U)$;

for any $u \in I_{\alpha}$ and $v \in I_{\beta}$, and any $j \leq n$,

$$
\frac{1}{k(j)} \xi\left(u, v, k(j), t_{j}\right) \leq F_{x_{\alpha(j)} x_{\beta(j)}}\left(t_{j}\right)+\frac{1}{j}
$$

and

$$
\frac{1}{q(j)} \xi\left(u, v, q(j), t_{j}\right) \geq F_{x_{\alpha(j)} x_{\beta(j)}}^{*}\left(t_{j}\right)-\frac{1}{j} .
$$

To do this, let $I_{0}$ and $I_{1}$ be disjoint compact subintervals of $\operatorname{int}(U)$, such that both $I_{0} \cap \omega$ and $I_{1} \cap \omega$ are infinite. Put $k(1)=1$ and $q(1)=2$ and assume by induction that $\left\{I_{\alpha} ; \alpha \in X_{n}\right\}, k(n)$ and $q(n)$ have been defined. Assume that $f^{j}\left(I_{\alpha}\right) \cap \omega$ is infinite whenever $j>r$ and $\alpha \in X_{n}$. Let $m$ be the period of $U$. By Lemma 5.3 we may assume that $x_{0}, x_{1} \in \operatorname{int}(U)$ and since $f \mid \omega$ is strongly transitive in $\operatorname{int}(U)$ (Theorem 3.7), there is an $s>\max \{r, q(n)\}$ such that $x_{0}, x_{1} \in \operatorname{int}\left(f^{m j}\left(I_{\alpha}\right)\right)$ whenever $\alpha \in X_{n}$ and $j \geq s$. Since $\omega$ is perfect (Theorem 3.1), it is easy to see that, for $i=0,1$ and any $\alpha \in X_{n}$, there is a point $a(\alpha, i) \in \operatorname{int}\left(I_{\alpha}\right)$ such that $f^{m s}(a(\alpha, i))=x_{i}$ and such that for any neighborhood $V$ of $a(\alpha, i), f^{m s}(V) \cap \omega$ is infinite.

Applying Lemma 5.1 we can find $q(n+1)>k(n+1)>m s$ and pairwise disjoint compact neighborhoods $I_{\beta}$ of the points $a(\alpha, i)$ for all $\beta \in X_{n+1}$, where $\beta=\alpha i$ (we use $\alpha i$ for the concatenation of $\alpha$ and $i$ ) such that (18)(22) are satisfied when $n$ is replaced by $n+1$.

Let $A=\bigcap_{n=1}^{\infty} \cup\left\{I_{\alpha} ; \alpha \in X_{n}\right\}$. Define a map, code: $A \rightarrow X$, by $\operatorname{code}(x)=$ $\alpha(1) \alpha(2) \cdots \alpha(n) \cdots$ if $x \in I_{\alpha(1) \alpha(2) \cdots \alpha(n)}$, for any $n$. Clearly code is a continuous map of $A$ onto $X$. Moreover, code is constant on each connected component $J(\alpha)=\bigcap_{n=1}^{\infty} I_{\alpha(1) \cdots \alpha(n)}$ of $A$; we have $\operatorname{code}(x)=\alpha$ for any $x \in J(\alpha)$. Thus if $A^{*} \subset A$ is a set that contains just one point from any connected component of $A$, then $A^{*}$ is a Borel set and code is a continuous one-to-one map from $A^{*}$ onto $X$. For $t \in T$, let $N_{t}=\left\{i \in N ; t_{i}=t\right\}$. Apply Lemma 5.4 to the decomposition $\left\{N_{t}\right\}_{t \in T}$ of $N$; let $B$ be the corresponding set. Then $\operatorname{code}^{-1}(B) \cap A^{*}$ is an uncountable Borel set, hence it contains a nonempty perfect subset $Q$ (cf., e.g., [8]).

Let $u, v \in Q, u \neq v$, let $\operatorname{code}(u)=\{\alpha(i)\}_{i=1}^{\infty}$ and let $\operatorname{code}(v)=\{\beta(i)\}_{i=1}^{\infty}$. By Lemma 5.4 there is an arbitrarily large $j$ such that $\alpha(j) \neq \beta(j)$ and $t=t_{j}$. Hence (21) gives $F_{u v}(t) \leq F_{x_{0} x_{1}}(t)$, and since $t$ is arbitrary in $T, F_{u v} \leq F_{x_{0} x_{1}}$. The argument for the second inequality in (17) is similar. And similarly, as in the proof of Lemma 5.2 (cf. the last paragraph of its proof), we see that $\omega_{f}(u) \subset \omega$ for any $u \in Q$. Since $Q=\bigcup_{i=1}^{\infty} f^{-i}(\omega) \cap Q$ (cf. (iv) of Theorem 3.1 ) there is an $n$ such that $f^{-n}(\omega) \cap Q$ is uncountable (and compact), and since $f$ is one-to-one on $Q$, there is a nonempty perfect subset $P$ of $f^{n}(Q) \cap \omega$. It is easy to verify that $P$ has all the required properties. 


\section{Proof of Theorems 2.3 and 2.4}

6.1 Lemma. Let $f \in C(I, I)$ and let $\left\{\omega_{i}\right\}_{i=1}^{\infty}$ be a sequence of distinct minimal periodic portions of maximal $\omega$-limit sets of $f$ of the second kind. If the periods of $\omega_{i}$ are bounded then $\lim _{i \rightarrow \infty} \operatorname{diam} \omega_{i}=0$.

Proof. Let $K_{i}$ be the convex hull of $\omega_{i}$, and let $m>0$ be a common multiple of the periods of all the $\omega_{i}$. Assume that the lemma is not true. Clearly, by replacing $f$ by $f^{m}$ if necessary, we may assume that $m=1$ and that, for any $i$, diam $\omega_{i}>\varepsilon$, where $\varepsilon$ is positive. By (iv) of Theorem 3.7 it suffices to consider the case when $K_{1}, K_{2}, \ldots$ is a strictly decreasing sequence.

Let $\delta>0$ be such that $\operatorname{diam} f(A)<\varepsilon$ for any set $A$ with $\operatorname{diam} A<\delta$. Let $K_{i}=\left[a_{i}, b_{i}\right]$. Then $a_{i}<a_{i+1}$ and $b_{i+1}<b_{i}$, for any $i$ (Theorem 3.7). Let $\omega^{0}=\left[a_{1}, a_{2}\right] \cap \omega_{1}$ and $\omega^{1}=\left[b_{2}, b_{1}\right] \cap \omega_{1}$. Since $\omega_{1}=\omega^{0} \cup \omega^{1}$ is indecomposable, one of the sets $\left[a_{1}, a_{2}\right],\left[b_{2}, b_{1}\right]$, say $\left[a_{1}, a_{2}\right]$, is mapped by $f$ over $\left[a_{2}, b_{2}\right]$. Thus $\left|a_{2}-a_{1}\right|>\delta$ and diam $\omega_{2}<\operatorname{diam} \omega_{1}-\delta$. By induction we get $\operatorname{diam} \omega_{i+1}<\operatorname{diam} \omega_{1}-i \delta$, for any $i$, which is impossible.

6.2 Lemma. Let $f \in C(I, I)$ and let $\left\{\omega_{i}\right\}_{i=1}^{\infty}$ be the minimal periodic portions of maximal $\omega$-limit sets of $f$ of the second kind. For any $i, j$, set $G_{i j}=$ $\inf \left\{F_{u v} ; u \in \omega_{i}\right.$ and $\left.v \in \omega_{j}\right\}$. Then

(i) Each $G_{i j}$ is zero on an interval $[0, \varepsilon(i, j)]$, where $\varepsilon(i, j)$ is a positive number.

(ii) The set $\left\{G_{i j} ; \omega_{i} \cap \omega_{j} \neq \varnothing\right\}$ has a finite number of minimal elements.

(iii) The set $\left\{G_{i i}\right\}_{i=1}^{\infty}$ has a finite number of minimal elements.

Proof. (i) By (v) of Theorem 3.7, there are distinct periodic points $p$ in $\omega_{i}$ and $q$ in $\omega_{j}$. Since $\min _{s} \delta_{p q}(s)=\varepsilon>0$, we have $F_{p q}(t)=0$, and hence, $G_{i j}(t)=0$, for $t \leq \varepsilon$, since $F_{p q} \geq G_{i j}$. Take $\varepsilon(i, j)=\varepsilon$.

(ii) We may assume that $\omega_{i} \neq \omega_{j}$, for any $i \neq j$. Let $K_{i}$ be the convex hull of $\omega_{i}$. Let $\varepsilon=\varepsilon(1,1)$ (cf. part (i)). We say that an $\omega_{i}$ is extremal, if $\operatorname{diam} \omega_{i}>\varepsilon / 2$ and if $K_{i}$ is properly contained in no $K_{j}$. By (iv) of Theorem 3.7 there are finitely many extremal $\omega_{i}$ 's. We may assume that $\omega_{1}, \ldots, \omega_{n(1)}$ are the extremal sets of $f$; note that $n(1) \geq 1$ since $f$ has positive topological entropy and hence at least one maximal $\omega$-limit set of the second kind (cf., e.g., [4]).

Let $m>0$ be an integer. We say that a set $\omega_{i}$ is significant if $\operatorname{diam} \omega_{i}>\varepsilon / 2$ and the period of $\omega_{i}$ is less than $m$. By Lemma 6.1, there are finitely many significant $\omega_{i}$ 's. Thus we may assume without loss of generality that there are integers $n(3) \geq n(2) \geq n(1)>0$ such that $\left\{\omega_{1}, \ldots, \omega_{n(2)}\right\}$ is the minimal system invariant under $f$ and containing all extremal and all significant sets, and that $\left\{\omega_{n(2)+1}, \ldots, \omega_{n(3)}\right\}$ is the system of all portions that have a point in common with some of $\omega_{i}, i \leq n(2)$ (cf. Theorem 3.7).

Note that $n(2)$ and $n(3)$, but not $n(1)$, depend on the parameter $m$. We show that the minimal elements of $\left\{G_{i j} ; \omega_{i} \cap \omega_{j} \neq \varnothing\right\}$ are in the set $M=$ $\left\{G_{i j} ; i, j \leq n(3)\right\}$ whenever $m$ is sufficiently large, depending on $n(1)$. Assume that $i>n(3)$ and that $\omega_{i} \cap \omega_{j} \neq \varnothing$. Then $\omega_{j}$ cannot be significant, hence $j>n(2)$. Let $u \in \omega_{i}$ and $v \in \omega_{j}$. It suffices to show that $F_{u v} \geq G$ for some $G$ in $M$. If $\operatorname{diam} f^{s}\left(\omega_{i} \cup \omega_{j}\right) \leq \varepsilon$ for any $s$ then $F_{u v}(t)=1$ for $t>\varepsilon$, and hence, $F_{u v} \geq G_{11}$.

So it suffices to consider the case when $\operatorname{diam} f^{s}\left(\omega_{i} \cup \omega_{j}\right)>\varepsilon$ for some $s$. 
Since the set $\left\{\omega_{1}, \ldots, \omega_{n(2)}\right\}$ is invariant, we may assume without loss of generality that $\operatorname{diam}\left(\omega_{i} \cup \omega_{j}\right)>\varepsilon$ and that

$$
\operatorname{diam} f^{s}\left(\omega_{i} \cup \omega_{j}\right) \leq \operatorname{diam}\left(\omega_{i} \cup \omega_{j}\right) \text { for any } s .
$$

Since one of the sets $\omega_{i}, \omega_{j}$, say $\omega_{i}$, has diameter $>\varepsilon / 2$, there is an extremal $\omega_{r}$ with $\omega_{i} \subset \operatorname{int}\left(K_{r}\right)$ (note that $\omega_{i}$ cannot be extremal since $i>n(1)$ ), and consequently, with $\omega_{i} \cup \omega_{j} \subset \operatorname{int}\left(K_{r}\right)$, since $\omega_{i} \cap \omega_{j} \neq \varnothing$ (cf. Theorem 3.7). By (v) of Theorem 3.7 and (iii) of Theorem 3.1, there are periodic points $a<b$ in $\omega_{r}$ with the property: If $J$ is an interval such that

$$
J \cap \omega_{r} \text { is finite, } \operatorname{diam} J>\varepsilon \text {, and } J \subset K_{r}
$$

then $J \subset(a, b)$. (It suffices to choose $a, b$ near the endpoints of $K_{r}$.)

Now $K_{i} \cap \omega_{r}$ is finite, since $f \mid \omega_{r}$ is strongly transitive in $\operatorname{int}\left(K_{r}\right)$ (cf. Theorem 3.7), and $K_{i} \cap \omega_{r}$ infinite would imply, that the minimal compact periodic interval containing $\omega_{i}$ must contain $K_{r}$, and hence, by Lemma 3.4, that $\omega_{i}=\omega_{r}$, which is impossible. Similarly $K_{j} \cap \omega_{r}$ is finite. Since $J=K_{i} \cup K_{j}$ satisfies (24), we have $\omega_{j} \cup \omega_{j} \subset(a, b)$. Take $t_{0}$ such that $\operatorname{diam}\left(\omega_{i} \cup \omega_{j}\right)<$ $t_{0}<b-a$. Let $F_{a b}\left(t_{0}\right)=\lambda_{r}$. Clearly $\lambda_{r}<1$. Let $\varepsilon(r, r)$ be as in (i). We have $G_{r r}(t)=0 \leq F_{u v}(t)$ for $t \leq \varepsilon(r, r)$, and by $(23), G_{r r}(t) \leq 1=F_{u v}(t)$ for $t>t_{0}$. And if $\varepsilon(r, r)<t_{0}$ then, by Lemma 4.1, $G_{r r}(t) \leq F_{a b}(t) \leq \lambda_{r}<F_{u v}(t)$ whenever $\varepsilon(r, r)<t \leq t_{0}$ and $m \geq n\left(\varepsilon(r, r), \lambda_{r}\right)$. Thus $G_{r r} \leq F_{u v}$ if $m \geq n\left(\varepsilon(r, r), \lambda_{r}\right)$.

It follows that when the parameter $m=\max \left\{n\left(\varepsilon(r, r), \lambda_{r}\right) ; 1 \leq r \leq n(1)\right\}$ then $\left\{G_{i j} ; i, j \leq n(3)\right\}$ contains the minimal elements of $\left\{G_{i j} ; \omega_{i} \cap \omega_{j} \neq \varnothing\right\}$.

(iii) It suffices to show that the minimal elements of $\left\{G_{i i}\right\}_{i=1}^{\infty}$ are contained in $\left\{G_{i i} ; i \leq n(2)\right\}$. But this follows from the above argument.

\subsection{Proof of Theorem 2.4. (A) follows by Theorem 2.2.}

To prove (B), let $\left\{\omega_{i}\right\}_{i=1}^{\infty}$ be the minimal periodic portions of all maximal $\omega$-limit sets of the second kind. (This system is nonempty since $f$ has positive topological entropy [16] and countable by Theorems 3.1 and 3.7.) For simplicity, denote by $\tilde{\omega}_{u}$ the maximal $\omega$-limit set containing $\omega_{f}(u)$.

(B1) Let $D=\left\{F_{u v} ; u\right.$ and $v$ are synchronous $\}$ and $E=\left\{F_{u v} ; u, v \in \omega_{i}\right.$ for some $i$. Clearly $D \supset E$. Conversely, let $F_{u v} \in D$. If $\tilde{\omega}_{u}\left(=\tilde{\omega}_{v}\right)$ is of the first kind then clearly

$$
\liminf _{n \rightarrow \infty} \delta_{u v}(n)=0
$$

and Theorem 2.1 implies $F_{u v}=\chi_{(0, \infty)} \in E$. If $\tilde{\omega}_{u}$ is of the second kind then by (iv) of Theorem 3.1 there is a $k$ such that $f^{k}(u), f^{k}(v)$ are in $\tilde{\omega}_{u}$, and hence, in some $\omega_{i}$. Thus $D=E$ and Lemma 6.2 gives the result on the spectrum $\Sigma(f)$.

Now let $D_{w}=\left\{F_{u v} ; u, v\right.$ satisfy $\left.(25)\right\}$ and $E_{w}=\left\{F_{u v} ; u \in \omega_{i}, v \in \omega_{j}\right.$ and $\omega_{i} \cap \omega_{j} \neq \varnothing$, for some $i$ and $\left.j\right\}$, and let $F_{u v} \in D_{w}$. Similarly, as before, one can easily verify that either $F_{u v}=\chi_{(0, \infty)}$ or there are $i, j, k$ such that $f^{k}(u) \in \omega_{i}$ and $f^{k}(v) \in \omega_{j}$, and (25) implies $\omega_{i} \cap \omega_{j} \neq \varnothing$. Thus $F_{u v} \in E_{w}$ and $D_{w} \subset E_{w}$.

Conversely, let $F_{u v} \in E_{w}$. Let $u \in \omega_{i}, v \in \omega_{j}$ and let $w \in \omega_{i} \cap \omega_{j}$. Take $Q=\{(u, v),(w, w)\}$ and apply Lemma 5.2 to get $x, y$ such that $F_{x y} \leq F_{u v}$ and $F_{x y}^{*}=\chi_{(0, \infty)}$. Hence $\liminf _{n \rightarrow \infty} \delta_{x y}(n)=0$ which implies $F_{x y} \in D_{w}$. Thus we have proved that $D_{w} \subset E_{w}$ and that $E_{w}$ has the lower bounds in 
$D_{w}$. Consequently, $E_{w}$ and $D_{w}$ must have the same system $\Sigma_{w}(f)$ of minimal elements and Lemma 6.2 gives the result. This proves (B1).

(B2) For any $k \leq m$ there are $x, y \in \omega_{i}$ for some $i$ such that $F_{x y}=F_{k}$ (see the proof of (B1)). Existence of $P_{k}$ now follows by Lemma 5.2 with $Q=\{(x, y),(x, x)\}$ and Lemmas 5.3 and 5.5.

(B4) Let any $u, v$ in a set $S$ satisfy (25). If for some $u \in S, \tilde{\omega}_{u}$ is of the first kind then similarly as in the proof of (B1) we get $F_{u v}=\chi_{(0, \infty)}$ for any $u, v \in S$ and $S=S_{0} \cup \varnothing$ is the corresponding decomposition.

Thus we may assume that $\tilde{\omega}_{u}$ is of the second kind, for any $u$ in $S$. Let $T_{i}=\left\{u \in S ; f^{j m(i)}(u) \in \omega_{i}\right.$, for some $\left.j\right\}$ where $m(i)$ is the period of $\omega_{i}$. By (iv) of Theorem 3.1, $S=\bigcup_{i=1}^{\infty} T_{i}$. We show that all but at most two of the sets $T_{i}$ are empty. Assume that $T_{j(1)}, T_{j(2)}, T_{j(3)}$ are nonempty. Let $d>0$ be a multiple of the periods of $\omega_{j(k)}$, and let $u(k)$ be in $T_{j(k)}$, for $k=1,2,3$. Since clearly $\liminf \operatorname{in}_{i \rightarrow \infty} \delta_{u(s) u(k)}(i d)=0$ whenever $s \neq k$, any two sets $\omega_{j(k)}$ must have a point in common. Hence by (iv) of Theorem 3.7, two of the sets, say $\omega_{j(2)}$ and $\omega_{j(3)}$ must coincide.

We have proved that $S=T_{j(1)} \cup T_{j(2)}$, for some $j(1)$ and $j(2)$. For any $u, v$ in $T_{j(1)}, F_{u v} \geq G_{j(1) j(1)} \geq F_{j}$, for some $j \leq m$, and similarly for $T_{j(2)}$. Thus $T_{j(1)} \cup T_{j(2)}$ is the decomposition of $S$ described in (B4). Moreover, it is easy to see that if $F_{u v}=F_{j}$ for any distinct $u, v$ in $S$, and if $j>m$, then $T_{j(1)}$ and $T_{j(2)}$ must be distinct and nonempty, and that each of the sets $T_{j(1)}, T_{j(2)}$ contains exactly one point.This proves (B3).

6.4 Proof of Theorem 2.3. This is a particular case of Theorem 2.4: For (i) see the proof of (B2), and for (ii) and (iii), the proofs of (B3) and (B4). Details are left to the reader.

\section{ACKNOWLEDGMENT}

Much of the work on this paper was done while the second author was a Visiting Professor at the University of Massachusetts, Amherst, in 1989 and 1990 and 1992. The support of this institution is gratefully acknowledged.

\section{REFERENCES}

1. L. S. Block and W. A. Coppel, One-dimensional dynamics, Lecture Notes in Math., vol. 1513, Springer-Verlag, Berlin, 1992.

2. R. Bowen, Topological entropy and Axiom A, Global Analysis, Proc. Sympos. Pure Math., vol. 14, Providence, R.I., 1970, pp. 23-41.

3. P. Collet and J.-P. Eckmann, Iterated maps on the interval as dynamical systems, Birkhäuser, Boston, Mass, 1980.

4. V. V. Fedorenko, A. N. Sharkovsky, and J. Smital, Characterizations of weakly chaotic maps of the interval, Proc. Amer. Math. Soc. 110 (1990), 141-148.

5. N. Franzová and J. Smítal, Positive sequence topological entropy characterizes chaos, Proc. Amer. Math. Soc. 112 (1991), 1083-1086.

6. Ch. K. Kenzhegulov and A. N. Sharkovsky, On properties of the set of limit points of an iterated sequence of a continuous function, Volž. Mat. Sb. 3 (1965), 343-348. (Russian)

7. M. Kuchta and J. Smítal, Two point scrambled set implies chaos, Proc. Europ. Conf. on Iteration Theory, Spain 1987, World Science, Singapore, 1989, pp. 427-430.

8. C. Kuratowski, Topologie, Vol. I, Polish Sci. Publ., Warsaw, 1958. 
9. T. Y. Li and J. A. Yorke, Period three implies chaos, Amer. Math. Monthly 82 (1975), 985-992.

10. M. Misiurewicz, Horseshoes for mappings of the interval, Bull. Acad. Polon. Sci. Ser. Math. 27 (1979), 167-169.

11. M. Misiurewicz and J. Smital, Smooth chaotic maps with zero topological entropy, Ergodic Theory and Dynamical Systems 8 (1988), 421-424.

12. Z. Nitecki, Topological dynamics on the interval, Ergodic Theory and Dynamical Systems. II, Proceedings (A. Katok, ed.), Birkhäuser, Boston, Mass, 1982, pp. 1-73.

13. D. Preiss and J. Smital, A characterization of non-chaotic maps of the interval stable under small perturbations, Trans. Amer. Math. Soc. 313 (1989), 687-696.

14. C. Preston, Iterates of piecewise monotone mappings on an interval, Lecture Notes in Math., vol. 1347, Springer-Verlag, Berlin, 1988.

15. A. N. Sharkovsky, The partially ordered system of attracting sets, Soviet Math. Dokl. 7 (1966), 1384-1386.

16. The behavior of a map in a neighborhood of an attracting set, Ukrain. Mat. Ž. 18 (1966), 60-83. (Russian)

17. $\ldots$ Continuous mapping on the set of $\omega$-limit sets of iterated sequences, Ukrain. Mat. Ž. 18 (1966), 127-130. (Russian)

18. B. Schweizer and A. Sklar, Probabilistic metric spaces, North-Holland, New York, 1983.

19. J. Smital, Chaotic functions with zero topological entropy, Trans. Amer. Math. Soc. 297 (1986), 269-281.

Department of Mathematics and Statistics, University of Massachusetts, Amherst, MASSACHUSETTS 01003

E-mail address: bert $@$ math. umass .edu

Institute of Mathematics, Comenius University, 842-15 Bratislava, Slovakia

Current address: Institute of Mathematics, Silesian University, 746-01 Opava, Czech Republic

E-mail address: jaroslav.smital@decsu.fpf.slu.cz 